\title{
FLEXIÓN Y DERIVACIÓN EN LA GRAMÁTICA FUNCIONAL DE DIK
}

JAVIER MARTIN ARISTA Universidad de La Rioja

\section{RESUMEN}

En este trabajo sostenemos que la morfología de la Gramática Funcional (GF) de Dik (1989) no puede considerarse unificada ni independiente, como resultado de la interacción de la morfología de la expresión con la del fondo léxico; y de la relevancia de la información recogida en el fondo léxico para la correcta operación de las reglas de expresión. De esta revisión de la morfología dikeana también se extrae la conclusión de que la GF tiene poder descriptivo suficiente para proporcionar la estructura subyacente de demostrativos pertenecientes a sistemas no binarios; los pronombres posesivos en inglés; las estructuras subyacentes de los términos que se obtienen por derivación morfológica; y la elipsis de algunos operandos. También se llega a la conclusión de que debe distinguirse entre reglas morfológicas de activación directa, y reglas de activación indirecta según éstas tengan o no reflejo directo en la expresión linguiística. 
INTRODUCCIÓN

En este trabajo nos ocupamos de la morfología en la Gramática Funcional (GF) de Dik (1989). ' Mantenemos la tesis de que, como resultado de la interacción de la morfología de la expresión con la del fondo léxico, y de la relevancia de la información de éste para las reglas de expresión, la morfología de la GF no puede considerarse unificada ni independiente. Fn el apartado 1 procedemos de un modo independiente de la teoría y hacemos notar la falta de un componente morfológico unificado. A continuación (apartado 2), nuestro análisis se lleva a cabo de manera dependiente de la teoría y nos referimos a los distintos tipos de reglas morfológicas, insistiendo en la interacción de las reglas morfológicas del fondo léxico (subapartado 2.1) con las del componente de expresion (subapartado 2.2). Finalmente, resumimos las aportaciones de este trabajo (apartado 3).

\section{LA AUSENCIA DE UN COMPONENTE MORFOLÓGICO UNIFICADO EN LA GF}

En este apartado revisamos de forma brevísima el estado de la cuestión en morfología desde una perspectiva independiente de las teorías aquí mencionadas. El trabajo de Nida (1970), para empezar, puede considerarse representativo de la morfología tradicional: en efecto, en una versión revisada de un trabajo de 1946, Nida lleva a cabo un análisis puramente descriptivo, que puede entroncarse en la tradición distribucionalista. ${ }^{2}$ Este autor trata los morfemas como unidades mínimas de la morfología e introduce criterios fonológicos en los principios para la identificación de morfemas (1970: 14). Sin embargo, el morfema fue rechazado como unidad mínima de la morfología en trabajos posteriores. Así, Chomsky (1965: 170) rechaz6 el morfema como base de la morfología flexiva y, más tarde, Aronoff (1976: 121) también descartó el morfema como base de la morfología derivativa. En general, los modelos posteriores, como la morfología de la palabra y el paradigma (Hockett 1954; Robins 1959; Matthews 1972), la morfología generativa (Chomsky 1965; Chomsky y Halle 1968; Aronoff 1976; Siegel 1979; Scalisse 1987) y la morfología léxica (Ki-

' La investigación de la que este trabajo es producto ha sido subvencionada por el Vicerrectorado de Investigación de la Universidad de La Rioja a través del proyecto 96PYB33JMA, titulado Aspectos metodológicos de la investigación lingüfstica en un paradigma funcional: sincronía y diacronía.

2 Para una visión en profundidad de los problemas planteados en este apartado, vid. VARELA ORTEGA (1990), Spencer (1992) y Matthews (1994). 
parsky 1982 y 1983; Katamba 1993) rechazan el morfema como unidad clave de la morfología y se concentran en la palabra.

Matthews (1972: 200), a quien podemos considerar representante de la morfología de la palabra y el paradigma, está en contra de la idea de un listado de formas básicas como estrato independiente y presta especial atención a la morfofonología y morfolexicología. Este lingüista es especialmente crítico respecto a la idea de una morfología basada en el morfema, tal y como se recoge en la herencia de los estructuralistas. Como Katamba (1993: 61) comenta:

The critique of morpheme-based approaches that this theory embodies (Word-and-Paradigm Morphology-J.M.) has contributed to a healthy examination of the nature of morphological representations in early years. WP morphology is critical of the somewhat naïve view found in some structuralist models of morphology (...) A theory of the morpheme that relies on the assumption that morphemes are always typified by a one-to-one pairing of morphemes with morphs is misguided.

Dentro de la corriente transformacional, que también se centra en la palabra —entendida como un predicado semántico abstracto en la semántica generativa-, no se contempla una morfología independiente. ${ }^{3}$ Como sucede con otros niveles de análisis, la morfología hace referencia a la sintaxis, pero no al contrario, dado el Principio de Autonomía de la Sintaxis. Como Scalisse (1987: 114) señala, la morfología de la Teoría Estándar (tal y como se recoge en The Sound Pattern of English) se ubica en componente morfologico, que forma parte, junto con el lexicón, del componente léxico. En el componente morfologico operan las reglas de formación de palabras, que son responsables de la composición y de la derivación; las reglas flexivas, que dan cuenta de la flexión; las reglas de ajuste, de naturaleza morfofonológica; y las convenciones que introducen los lindes externos (los lindes internos son introducidos por las reglas de formación de palabras).

La morfología generativa ha recibido dos críticas principales: primero, se ha criticado la falta de dimensión paradigmática de la teoría, carencia motivada, sin lugar a dudas, por la prioridad sintagmática de la lingüística chomskiana. Como indica Marle (1985: 9), que distingue productividad de creatividad léxica y situa ambas en el lexicón (con especial énfasis en las relaciones paradigmáticas), within the framework of morphological theories of the format of

3. Sobre la morfología de la Teoría Estándar, vid. Siegel (1979: 101), MatTHEws (1974: 216) y KATAMBA (1993: 5). WATTERS (1985: 86) compara la morfología del modelo de ChOMSKY (1981) con la de la GF. 
Aronoff (1976-J.M.), the field of paradigmatic structure proper is largely neglected. En segundo lugar, la morfología léxica ha surgido como reacción al excesivo papel desempeñado por los lindes en la morfología generativa. En la morfología léxica, una estructura jerárquica compuesta por distintos estratos justifica la diferencia de comportamiento entre unos afijos y otros, de manera que distintos afijos se introducen en distintos estratos. En cada uno de estos estratos, una regla fonológica acompaña a cada regla morfológica (Katamba 1993), lo que implica que la morfología tampoco es independiente.

Tampoco existe una morfología independiente para Bauer (1988: 89), quien considera a la palabra la unidad morfológica y sitúa la afijación en una posición central dentro de la morfología. Otros procesos, como la composición y la flexión, ocupan posiciones periféricas que limitan con la fonología y la sintaxis.

En resumen, de este breve repaso al estado de la cuestión en morfología se desprenden las siguientes conclusiones: (i) el morfema ha sido abandonado como unidad clave de la morfología en favor de la palabra; (ii) la morfología constituye un componente o subcomponente unificado aunque no independiente, puesto que debe hacer referencia, como mínimo, a la sintaxis y a la fonología; (iii) existe consenso respecto a que la morfología debe ocuparse de procesos como la composición, la derivación y la flexión; (iv) la morfología debe definirse dentro del contexto de la teoría, utilizando la terminología y el aparato descriptivo del modelo, puesto que la comparación entre los formalismos de distintos modelos resulta casi imposible, a menos que nos limitemos a cuestiones muy generales como las aquí apuntadas. ${ }^{4}$

Procedamos a examinar la morfología en el marco de la GF. Primeramente, destaca el hecho de que, como el propio Dik (1989: 297) indica, no existe un componente morfológico unificado en la GF:

Morphological structure is not considered to be a unified phenomenon in FG: there is no single component in the grammar which deals with all and only the word-internal grammatical structures. Rather, the information which may finally be mapped into the structure of words is judged to spring from different sources, and what is expressed morphologically (synthetically) within a word might as well have been expressed syntactically (analytically) through a combination of words.

Dik justifica la falta de unidad de la morfología con el hecho de que una morfología (o estructura interna de las palabras) unificada supondría un trata-

4 Vid. ANDERSON (1982:572). 
miento distinto de la sintaxis (o estructura externa de las palabras), con lo que la teoría perdería poder generalizador y, en definitiva, grado de adecuación tipológica: en efecto, una categoría morfológica puede ser resultado de la derivación en una lengua y resultado de la flexión en otra. En consecuencia, la estructura interna de las palabras puede ser generada por alguno, o por una combinación, de estos componentes:

(i) El lexicón, que contiene una lista de todas las formas que no es posible producir por medio de una regla productiva en la sincronía. Estas formas se hacen inmediatamente disponibles por una regla de prioridad léxica.

(ii) El fondo léxico, que produce predicados derivados como manager a partir de predicados básicos como manage. Las reglas de formación de predicados (y términos) son responsables de la composición y de la derivación de unos predicados (y términos) de otros, por medio de la introducción de los operadores de predicados (y términos) pertinentes.

(iii) El componente de expresión, que asigna la forma a las expresiones lingüísticas subyacentes.

A modo de sumario, y siguiendo a Watters (1985: 89), diremos que la morfología derivativa es un producto del fondo léxico, en tanto que la morfología flexiva tiene lugar en el componente de expresión: Inflexional morphology is what is relevant to the expression rules (...) Derivational morphology is what is relevant to the fund.

Otro dato destacable es que la GF sustenta una morfología basada en las raíces de las entradas léxicas. Como Dik (1989: 296) indica, there is reason to assume that the basic form of the predicate is always a stem, even though it may coincide with one or more forms which can also be used as words. En efecto, Dik establece en el principio de prioridad léxica que debe seleccionarse una palabra siempre que esté disponible para su uso inmediato en el lexicón; si lo que hay disponible es una raíz, debe aplicarse a la raíz la regla necesaria en el componente de expresión; y si no existe ni una ni otra, debe aplicarse la regla de derivación de predicados pertinente al predicado en el fondo léxico. La elección de la raíz léxica como unidad clave de la descripción morfológica está justificada por la crítica de la que es susceptible la elección de la palabra: la palabra como unidad de descripción en morfología es una elección que parece hecha a medida de una lengua con una morfología muy erosionada como el inglés, pero parece más difícil de sostener al analizar lenguas morfológicamente más complejas como las lenguas clásicas o el alemán. 


\section{Tipos de REglas MORFológicas en la GF}

Dados los dos subcomponentes morfológicos situados en el fondo léxico y en el componente de expresión, que se encargan de la derivación y de la flexión respectivamente, veamos qué tipo de reglas operan en cada uno de estos dos subcomponentes.

\subsection{Reglas morfológicas del fondo léxico}

La GF adopta una posición intermedia respecto a la controversia de la productividad en el componente léxico; como Dik (1980: 27) concluye, ni la producción de todas las entradas léxicas derivadas por medio de reglas ni el almacenamiento de toda entrada no básica en el lexicón pueden dar cuenta satisfactoriamente de la productividad léxica:

Given the entrance of some new count noun into the lexicon of Dutch, such as wokkel in the sense of «type of biscuit», we can immediately predict the correctness of the diminutive wokkeltje in the sense of «little wokkel». Listing wokkeltje in the lexicon is not only superfluous, it also does not capture the ability of speakers of Dutch to form correctly the diminutive on the basis of the basic nominal. Predicate formation rules are meant to reflect this ability.

De esta manera, se justifica la independencia de las reglas de formación de predicados y su inclusión en el fondo léxico, puesto que proporcionan marcos predicativos igual que el lexicón, si bien los almacenados en el lexicón son básicos y los producidos en el fondo léxico son derivados. Esta distinción también se aplica a los términos, que se almacenan en el lexicón cuando son básicos y se producen en el fondo léxico por medio de reglas de formación de términos cuando se trata de términos derivados. Pasamos a ocuparnos de los procesos morfológicos asociados con la formación de predicados y con la formación de términos.

\subsubsection{Reglas de formación de predicados}

Las reglas de formación de predicados no sólo contienen procesos morfológicos como la composición y la derivación; también dan cabida a procesos que se manifiestan de forma analítica, esto es, sintácticamente. Como 
ejemplo de regla de formación de predicados que se expresa analíticamente por medio de varias palabras, Dik (1980: 53) propone la construcción causativa en holandés:

(1) Formación de predicados causativos en holandés input: un marco predicativo cualquiera $f\left(x_{1}\right) \ldots\left(x_{n}\right)$

output: laten $_{v} f\left(x_{0}\right)_{A g}\left(x_{1}\right) \ldots\left(x_{n}\right)$

donde $\left(\mathrm{x}_{0}\right)$ representa una nueva posición argumental en la que se introduce el término que recibe la función semántica Causante.

Esta regla produce marcos predicativos derivados a partir de marcos predicativos básicos. Dado un marco predicativo como el de geven, dar, en (2.a), la regla produce un marco predicativo derivado como el descrito en (2.b):

(2)

a. geven $_{\mathrm{v}}\left(\mathrm{x}_{1} \text { : <animado }>\left(\mathrm{x}_{1}\right)\right)_{\mathrm{Ag}}\left(\mathrm{x}_{2}\right)_{\mathrm{PaC}}\left(\mathrm{x}_{3}:<\text { animado }>\left(\mathrm{x}_{3}\right)\right)_{\mathrm{Rec}}$

b. laten geven $_{\mathrm{v}}\left(\mathrm{x}_{0}\right)_{\mathrm{Ag}}\left(\mathrm{x}_{1} \text { : <animado> }\left(\mathrm{x}_{1}\right)\right)_{\mathrm{Ag}}\left(\mathrm{x}_{2}\right)_{\mathrm{Pac}}\left(\mathrm{x}_{3}\right.$ : <animado> $\left.\left(\mathrm{x}_{3}\right)\right)_{\mathrm{ReC}}$

Si insertamos términos en las posiciones argumentales de los marcos predicativos del ejemplo anterior, obtenemos expresiones lingüísticas como éstas (Dik 1980: 55):

a. Marie gaf een kado aan Karel

Marie dio un regalo a Karel

«Marie le hizo un regalo a Karel»

b. Piet liet Marie een kado aan Karel geven

Piet hizo a Marie un regalo a Karel dar

«Piet hizo que Marie le hiciera un regalo a Karel»

Otro proceso que se manifiesta sintácticamente, además de la extensión del número de argumentos del marco predicativo de entrada que acabamos de mencionar, es la reducción del número de argumentos del marco predicativo de entrada. El cambio de función sintáctica de los argumentos del marco predicativo de entrada puede manifestarse tanto sintáctica como morfológicamente. Como procesos morfológicos (casi) puros tenemos la modificación del predicado de entrada y el cambio de categoría léxica del predicado de entrada. Ambos procesos, como vamos a ver, están íntimamente relacionados, de tal forma que el cambio de categoría léxica del predicado de entrada im- 
plica, frecuentemente, el cambio de forma y viceversa. Un predicado básico nominal como golfo puede dar lugar a un predicado derivado de carácter verbal como golfear. En este caso, el cambio de forma implica el cambio de función y viceversa. En otros casos, como el del predicado adjetival último, no se precisa un cambio de forma para el cambio de categoría léxica:

(4)

a. El último tren sale a las doce

b. El último que cierre la puerta

El cambio de categoría léxica adjetivo-pronombre se manifiesta en la sintagmática y no acarrea cambio de forma, esto es, se produce mediante la derivación por morfema cero.

El output de las reglas de formación de predicados constituye el input de las reglas de expresión, que asignan la forma correcta al predicado derivado. Sean las reglas de formación de predicados de formación de agentes nominales y de negación de predicados adjetivales en inglés:

(5)

a. Formación de agentes nominales

lie-liar teach-teacher interrogate-interrogator

b. Negación de predicados adjetivales comfortable-uncomfortable delicate-indelicate similar-dissimilar

En ambos casos, el componente de expresión introduciría el afijo con el que los predicados aparecen en las expresiones lingüísticas en la sincronía. En (5.b) no existe cambio de categoría léxica, pero sí de forma del predicado. En (5.a), por el contrario, la regla de agentes nominales produce predicados nominales derivados a partir de predicados verbales básicos. La posibilidad de explicar estos fenómenos por medio de una operación de cambio de categoría, de función o de estructura podría no ser compatible con la exclusión de las transformaciones del aparato descriptivo de la teoría. En consecuencia, es necesario redefinir esta exclusion (Dik 1980: 51):

Predicate formation rules (...) may have certain structure-changing, that is transformational properties, in that the output predicate- 
frame of a predicate formation rule may consist of a restructuring of the input predicate-frame(s), involving re-allocation of a semantic function, loss (or addition) of argument positions, and shifts in semantic aspects. It is clear, then that the general ban on structure-changing operations characteristic of FG will have to be relaxed as far as these properties of the predicate formation component are concerned.

Este nuevo enfoque es el resultado del desplazamiento de la complicación del aparato teórico al componente léxico, reflejo, a su vez, de la prioridad metodológica dada a la semántica. $O$, dicho de otra manera, es preferible que no haya transformaciones, pero, si debe haberlas, éstas deben tener lugar en el fondo léxico para que la sintaxis tenga que hacer referencia a la semántica pero no al contrario.

\subsubsection{Reglas de formación de términos}

En el fondo léxico encontramos tanto predicados como términos. Estos últimos son expresiones lingüísticas que se usan para referirse a entidades o conjuntos de entidades de un mundo (Dik 1989: 55). A pesar de la distinción inicial, predicados y términos están estrechamente vinculados puesto que las estructuras subyacentes de las frases de términos se construyen por medio de predicados. ${ }^{5}$ Entre los términos básicos, los que están almacenados en el lexicón, encontramos los pronombres personales, los nombres propios, los días de la semana, los meses del año, los puntos cardinales, los signos del zodíaco, etc.; los términos derivados, que son la gran mayoría, se producen en el fondo léxico por medio de reglas de formación de términos. Dichas reglas son capaces de producir frases nominales tan complejas como (6):

(6) El coche que no me compré cuando estaba de oferta pero recomendé a mi amigo Luis

Como sucede en el caso de (6), la mayor parte de los términos derivados se producen por medio de procedimientos sintácticos: por medio de operado-

5 Rijkhoff (1990) ha propuesto un análisis unificado de frases de términos y predicaciones, de tal forma que exista una estructura jerárquica de los términos que distinga entre términos calificados (o terminos nucleares), términos cuantificados y términos completos (o términos expandidos). Esta jerarquía es semejante a la propuesta para los enunciados (Hengeveld 1990). Sobre la estructura subyacente de los términos, vid. VOOGT-VAN ZUTPHEN (1989: 164); y acerca de los operadores de términos, vid. BROWN (1985: 127). 
res de términos. ${ }^{6}$ Así, como acabamos de ver, dado un predicado básico como coche, podemos generar un término derivado complejo que se manifiesta en la sintagmática como una cláusula de relativo. Dicho término derivado complejo se inserta a continuación en una de las posiciones argumentales de un marco predicativo dado, como la de Paciente en (7.a), dando lugar a una expresión lingüística como (7.b):

a. comprar $_{v}$ (d1xi: MaríaN (xi))Ag (el coche que no me compré cuando estaba de oferta pero recomendé a mi amigo Luis) ${ }_{\mathrm{Pac}}$ )

b. María compró el coche que no me compré cuando estaba de oferta pero recomendé a mi amigo Luis

Otros operadores de términos garantizan la distinción definido (d)/indefinido (i). Una vez insertado un operador u otro, las reglas del componente de expresión producen la forma correcta, no sólo desde el punto de vista de la información pragmática y espacial de los interlocutores, sino también desde la perspectiva de la concordancia con el operando. Este mecanismo se aprecia más nítidamente en lenguas que, como el español, distinguen género y número en el artículo (el operador de género lo introducimos en la discusión de los pronombres personales; mientras tanto, debe entenderse que los artículos y demostrativos en español también son producto de la actuación del operador de género $\mathbf{M} / \mathbf{F})$ :

a. $\left(\mathbf{d} 1 \mathrm{x}_{\mathrm{i}}: \operatorname{libro}_{\mathrm{N}}\left(\mathrm{x}_{\mathrm{i}}\right)\right)=$ el libro

b. $\left(\mathbf{i} 1 \mathrm{x}_{\mathrm{i}}:\right.$ libro $\left._{\mathrm{N}}\left(\mathrm{x}_{\mathrm{i}}\right)\right)=$ un libro

c. $\left(\operatorname{dmx}_{\mathrm{i}}: \operatorname{libro}_{\mathrm{N}}\left(\mathrm{x}_{\mathrm{i}}\right)\right)=$ los libros

d. $\left(\operatorname{imx}_{\mathrm{i}}: \operatorname{libro}_{\mathrm{N}}\left(\mathrm{x}_{\mathrm{i}}\right)\right)=$ unos libros

La ventaja de este tratamiento por medio de operadores de términos es que ciertas distinciones, como las deícticas, ya se incluyen en el fondo léxico, puesto que los operadores posibilitan la referencia constructiva si se trata de un referente que no está disponible en la información perceptual o pragmática del receptor; y la referencia identificativa si se trata de un referente accesible en dicha información (Dik 1989: 139).

- Para un tratamiento funcional de los fenómenos de determinación y cuantificación, vid. Moreno Cabrera (1991: 227), Downing y Locke (1992: 419) y Givón (1993 vol I: 80f). 
El operador de términos definido/indefinido interactúa con el operador específico (e) y genérico $(\mathrm{g})$ :

a. $\left(\right.$ ilex $\left._{i}: \operatorname{libro}_{N}\left(x_{i}\right)\right)=$ un libro

Hay un libro en la mesa

b. $\left(i \lg x_{i}\right.$ : $\left.\operatorname{libro}_{N}\left(x_{i}\right)\right)=$ un libro

Un libro es la mejor compañía

Los demostrativos también se captan por medio de operadores de términos, lo que sitúa todo el fenómeno de la deixis en parámetros pragmáticos y/o perceptuales, esto es, como una consecuencia de la presencia o ausencia de una información pragmática del receptor. En el caso de los demostrativos, seleccionamos el operador de términos de lejanía (rem) o proximidad (prox), el cual, a su vez, activa la regla de expresión correspondiente que garantiza la concordancia formal de (género, número y/o caso) en lenguas que cuentan con un sistema de demostrativos sensible a la forma del sustantivo, como sucede en español:

(10)

a. $\left(\right.$ d 1 pro $\left.x_{i}: \operatorname{libro}_{N}\left(x_{i}\right)\right)=$ este libro

b. $\left(\mathrm{d} 1\right.$ rem $\left.x_{i}: \operatorname{libro}_{N}\left(x_{i}\right)\right)=$ aquel libro

En español se plantea el problema de que un operador binario como proximidad/lejanía no puede generar, tras la actuación de las reglas de expresión, una expresión como:

\section{(11) Ese libro no es mío}

Sería necesario introducir un tercer operador que hiciera referencia a aquellas entidades que no se encuentran ni próximas ni remotas, un operador que podríamos llamar de cercanía (cer):

a. $\left(\mathrm{d} 1\right.$ cer $\mathrm{x}_{\mathrm{i}}$ : libro $\left.\mathrm{N}_{\mathrm{N}}\left(\mathrm{x}_{\mathrm{i}}\right)\right)=$ ese libro

b. $\left(\right.$ dmcer $_{x_{i}}$ : $\left.\operatorname{libro}_{N}\left(x_{i}\right)\right)=$ esos libros

Los cuantificadores y numerales también se introducen por medio de operadores de términos: 
(13)

a. (i $15 x_{\mathrm{i}}$ : libro $_{\mathrm{N}}\left(\mathrm{x}_{\mathrm{i}}\right)$ ) = quince libros

b. (i $3 / 15 x_{i}$ : libro ${ }_{N}\left(x_{i}\right)$ ) $=$ tres de los quince libros

c. $\left(i 3 /\right.$ dn $x_{i}$ : $\left.\operatorname{libro}_{N}\left(x_{i}\right)\right)=$ tres de los libros

d. (i muchos/dn $x_{i}$ : libro $_{N}\left(x_{i}\right)$ ) = muchos de los libros

e. (i algunos/dn $x_{i}$ : libro ${ }_{N}\left(x_{i}\right)$ ) $=$ algunos de los libros

f. $\left(d\right.$ todos/dn $x_{i}$ : libro $\left.{ }_{N}\left(x_{i}\right)\right)=$ todos los libros

g. $\left(\mathrm{d}^{2} \mathrm{x}_{\mathrm{i}}\right.$ : $\left.\operatorname{libro}_{\mathrm{N}}\left(\mathrm{x}_{\mathrm{i}}\right)\right)=$ el primer libro

h. $\left(\right.$ d $21^{2} x_{i}$ : libro $\left._{N}\left(x_{i}\right)\right)=$ los dos primeros libros

i. (d 1 siguiente ${ }^{2} x_{i}$ : libro $\left.{ }_{N}\left(x_{i}\right)\right)=$ el siguiente libro

j. (d 1 siguiente $/ \mathrm{d}^{\mathrm{O}} 10 \mathrm{x}_{\mathrm{i}}$ : libro $_{\mathrm{N}}\left(\mathrm{x}_{\mathrm{i}}\right)$ ) $=$ el siguiente de los diez libros

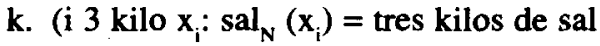

Los pronombres personales, tradicionalmente discutidos en el campo de la flexión nominal, se incluyen aquí como términos básicos (Dik 1989: 131):

$\left(\mathrm{d} 1 \mathrm{x}_{\mathrm{i}}:[+\mathrm{E},-\mathrm{R}]\left(\mathrm{x}_{\mathrm{i}}\right)\right)$

$\left(d 1 x_{i}:[-E,+R]\left(x_{i}\right)\right)$

$\left(d 1 x_{i}:[-E,-R,+M]\left(x_{i}\right)\right)$

$\left(d 1 x_{i j}:[-E,-R,+F]\left(x_{i}\right)\right)$

$\left(d m x_{i}:[+E,-R]\left(x_{i}\right)\right)$

$\left(d m x_{i}:[-E,+R]\left(x_{i}\right)\right)$

$\left(d m x_{i}:[-E,-R]\left(x_{i}\right)\right)$

$\begin{array}{ll}\text { +Sujeto } & \text {-Sujeto } \\ \text { I } & \text { me } \\ \text { you } & \text { you } \\ \text { he } & \text { him } \\ \text { she } & \text { her } \\ \text { we } & \text { us } \\ \text { you } & \text { you } \\ \text { they } & \text { them }\end{array}$

En (14), d significa definido; 1 y $\mathrm{m}$ singular y plural respectivamente; $M$ y F son la abreviatura de masculino y femenino; y $E$ y $R$ representan a los participantes (emisor y receptor). Aunque Dik no los formaliza, dada la regla de formación de predicados a partir de términos por medio de la asignación de una función semántica al término de entrada (Dik 1989: 173), a los pronombres se les puede asignar las siguientes estructuras subyacentes:

$\begin{array}{ll}\left(\mathrm{d} 1 \mathrm{x}_{\mathrm{i}}:[+\mathrm{E},-\mathrm{R}](\mathrm{xi})\right)_{\mathbf{P o s}}= & \text { mine } \\ \left(\mathrm{d} 1 \mathrm{x}_{\mathrm{i}}:[-\mathrm{E},+\mathrm{R}](\mathrm{xi})\right)_{\mathbf{P o s}}= & \text { yours } \\ \left(\mathrm{d} 1 \mathrm{x}_{\mathrm{i}}:[-\mathrm{E},-\mathrm{R},+\mathrm{M}]\left(\mathrm{x}_{\mathrm{i}}\right)\right)_{\mathbf{P o s}}= & \text { his } \\ \left(\mathrm{d} 1 \mathrm{x}_{\mathrm{i}}:[-\mathrm{E},-\mathrm{R},+\mathrm{F}]\left(\mathrm{x}_{\mathrm{i}}\right)\right)_{\mathbf{P o s}}= & \text { hers } \\ \left(\operatorname{dmx}_{\mathrm{i}}:[+\mathrm{E},]\left(\mathrm{x}_{\mathrm{i}}\right)\right)_{\mathbf{P o s}}= & \text { ours }\end{array}$




$$
\begin{array}{ll}
\left(\operatorname{dmx}_{\mathrm{i}}:[-\mathrm{E},+\mathrm{R}]\left(\mathrm{x}_{\mathrm{i}}\right)\right)_{\mathrm{Pos}}= & \text { yours } \\
\left(\mathrm{dmx}_{\mathrm{i}}:[-\mathrm{E},-\mathrm{R}]\left(\mathrm{x}_{\mathrm{i}}\right)\right)_{\mathrm{Pos}}= & \text { theirs }
\end{array}
$$

En este caso, la función semántica Poseedor asignada a la estructura subyacente de los pronombres personales produce los pronombres posesivos. Puesto que la reasignación de categoría léxica debe tener lugar en el fondo léxico, diremos que la estructura subyacente de los adjetivos posesivos, que también deben tratarse como términos, se encuentra en el fondo léxico, donde la categoría léxica adjetivo reemplaza a la categoría pronombre; y se almacenan las representaciones fonologicas de los adjetivos posesivos como términos básicos:
a. $\left(\mathrm{d} 1 \mathrm{x}_{\mathrm{i}}:[+\mathrm{E},-\mathrm{R}]_{\text {Pron }}\left(\mathrm{x}_{\mathrm{i}}\right)\right)_{\text {Pos }}>\left(\mathrm{d} 1 \mathrm{x}_{\mathrm{i}}:[+\mathrm{E},-\mathrm{R}]_{\mathrm{Adj}}\left(\mathrm{x}_{\mathrm{i}}\right)\right)_{\text {Pos }}=\mathrm{my}$
b. $\left(\mathrm{dl}_{\mathrm{i}}:[-\mathrm{E},+\mathrm{R}]_{\text {Pron }}\left(\mathrm{x}_{\mathrm{i}}\right)\right)_{\text {Pos }}>\left(\mathrm{d} 1 \mathrm{x}_{\mathrm{i}}:[-\mathrm{E},+\mathrm{R}]_{\mathrm{Adj}}\left(\mathrm{x}_{\mathrm{i}}\right)\right)_{\text {Pos }}=$ your
c. $\left(\mathrm{d} 1 \mathrm{x}_{\mathrm{i}}:[-\mathrm{E},-\mathrm{R},+\mathrm{M}]_{\text {Pron }}\left(\mathrm{x}_{\mathrm{i}}\right)\right)_{\text {Pos }}>\left(\mathrm{d} 1 \mathrm{x}_{\mathrm{i}}:[-\mathrm{E},-\mathrm{R},+\mathrm{M}]_{\mathrm{Adj}}\left(\mathrm{x}_{\mathrm{i}}\right)\right)_{\text {Pos }}=$ his
d. $\left(\mathrm{d} 1 \mathrm{x}_{\mathrm{i}}:[-\mathrm{E},-\mathrm{R},+\mathrm{F}]_{\text {Pron }}\left(\mathrm{x}_{\mathrm{i}}\right)\right)_{\text {Pos }}>\left(\mathrm{d} 1 \mathrm{x}_{\mathrm{i}}:[-\mathrm{E},-\mathrm{R},+\mathrm{F}]_{\mathrm{Adj}}\left(\mathrm{x}_{\mathrm{j}}\right)\right)_{\mathrm{Pos}}=$ her
e. $\left(\mathrm{dmx}_{i}:[+\mathrm{E},]_{\text {Pron }}\left(\mathrm{x}_{\mathrm{i}}\right)\right)_{\text {Pos }}>\left(\mathrm{dmx}_{\mathrm{i}}:[+\mathrm{E},]_{\mathrm{Adj}}\left(\mathrm{x}_{\mathrm{i}}\right)\right)_{\text {Pos }}=$ our
f. $\left(\mathrm{dmx}_{\mathrm{i}}:[-\mathrm{E},+\mathrm{R}]_{\text {Pron }}\left(\mathrm{x}_{\mathrm{i}}\right)\right)_{\text {Pos }}>\left(\mathrm{dmx}_{\mathrm{i}}:[-\mathrm{E},+\mathrm{R}]_{\mathrm{Adj}}\left(\mathrm{x}_{\mathrm{i}}\right)\right)_{\mathrm{Pos}}=$ your
g. $\left(\mathrm{dmx}_{\mathrm{i}}:[-\mathrm{E},-\mathrm{R}]_{\text {Pron }}\left(\mathrm{x}_{\mathrm{i}}\right)\right)_{\text {Poss }}>\left(\mathrm{dmx}_{\mathrm{i}}:[-\mathrm{E},-\mathrm{R}]_{\mathrm{Adj}}\left(\mathrm{x}_{\mathrm{i}}\right)\right)_{\text {Poss }}=$ their

Por último, los términos pueden ser objeto de una pregunta, como en (17.a) y (17.b); de una relativización, como en (17.c) y (17.d); y de una relación anafórica, como en (17.e) y (17.f). Estas tres operaciones se indican con los operadores de términos Q, R y A respectivamente (Dik 1989: 160):

\section{(17)}

a. $\left(\mathbf{Q x _ { i }}:<\right.$ humano $\left.>\left(\mathbf{x}_{\mathrm{i}}\right)\right)=$ who?

b. $\left(\mathbf{Q} \mathbf{x}_{\mathrm{i}}\right.$ : <inanimado $\left.>\left(\mathbf{x}_{\mathrm{i}}\right)\right)=$ what?

c. $\left.\left(\text { d l }_{\mathrm{i}}: \operatorname{man}_{\mathrm{N}}\left(\mathrm{x}_{\mathrm{i}}\right) \text { : steal } \mathrm{v}_{\mathrm{v}}\left(\mathbf{R x _ { i }}\right)_{\mathrm{Ag}} \text { (the car }\right)_{\mathrm{Pac}}\right)=$ the man who stole the car

d. $\left.\left(d 1 x_{j} \text { : hurricane }{ }_{N}\left(x_{i}\right): \text { ruin }_{v}\left(R_{x_{j}}\right)_{\text {Fuerza }} \text { (the house }\right)_{P a c}\right)=$ the hurricane that ruined the house

e. introduce $\left.{ }_{v}\left(d_{1} x_{i}: \text { Peter }_{N}\left(x_{i}\right)\right)_{A g}\left(A x_{i}\right)_{P_{a c}}\right)=$ Peter introduced himself

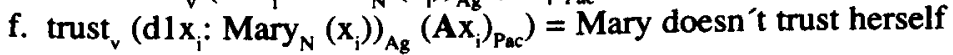

Hasta aquí, hemos tratado las reglas de formación de términos que, si bien se manifiestan formalmente tras la actuación de las reglas de expresión, tienen su origen en distinciones semánticas y/o pragmáticas que se establecen en el fondo léxico. El fondo léxico, sin embargo, también debe generar las estructuras subyacentes de los términos que se obtienen por derivación morfológica. Entre estos términos, podríamos contar los siguientes ejemplos: 


\section{(18)}
a. dominguero
b. marxismo
c. chomskiano
d. sureño

El caso de (18.a) no presenta mayores problemas, puesto que se trata de derivar un término derivado de otro básico por medio de la regla de formación de agentes nominales, como en carpintero o cartero. El ejemplo en (18.b) tampoco ofrece dificultades, puesto que, como sucede en (18.a), la operación de la regla de derivación de términos no supone una transposición léxica. En (18.c) y (18.d) sí que se da dicha transposición, ya que la regla deriva un término adjetival de uno nominal. La transposición también tiene lugar en ejemplos como:

\section{a. Me gusta esta casa \\ b. Me gusta ésta}

Para que el aparato descriptivo de la GF produzca la expresión lingüística en (19.b) es necesaria una regla de elipsis del operando, cuyo resultado sea que el operador de términos se convierta en operando, con la correspondiente transposición adjetivo-pronombre. De nuevo, lo que se manifiesta en la sintagmática es reflejo de una opción de tipo contextual entre la repetición o la elipsis del predicado nominal. En resumen, la transposición de predicado pronominal a predicado adjetival y viceversa requiere regla de elipsis del operando (adjetivos-pronombres demostrativos en inglés y español) y/o cambio de forma (adjetivos-pronombres posesivos en inglés); otros casos de transposición, como la transposición pronombre-nombre de she y he, no requieren elipsis del operando ni cambio de forma:
A: Was it a she?
B: No, it was a he

Hasta aquí hemos mantenido la distinción ortodoxa entre la derivación, que tiene lugar en el fondo léxico, y la flexión, que se produce en el componente de expresión. Esta distinción ha sido puesta en duda por De Groot (1990: 200 ), quien ha observado que hay morfemas que actúan como el resultado de la derivación en unos casos y de la flexión en otros: 
(21)

a. The shooting of the hunter

b. The hunter is shooting

Efectivamente, la aplicación del morfema -ing en los ejemplos anteriores del inglés, es resultado de la derivación en (21.a) y de la flexión en (21.b). Dada la dificultad de mantener la distinción entre lo puramente derivativo (que se produce en el fondo léxico) y lo exclusivamente flexivo (que se articula en el componente de expresión) se propone el almacenamiento en el lexicón de todos los morfemas de una lengua, tanto libres como trabados, que son de este modo tratados como entradas léxicas básicas, esto es, como predicados y términos básicos (De Groot 1990: 201):

Although Lexicon, Predicate formation and Expression rules are considered to be distinct components of the grammar, they do not necessarily contain their own list of (grammatical) elements. I suggest that all elements, both predicates and grammatical elements are contained in the Lexicon. Triggers which arise through Predicate formation and Expression rules components may penetrate the Lexicon and pick out formal elements. Placement rules account for the positions these elements have in the linguistic expressions.

Esta modificación del modelo ortodoxo, que implica un desplazamiento de los morfemas flexivos al lexicón, supone una reducción aún mayor del componente de expresión, que queda así limitado a la aplicación de las reglas fonológicas y de una sintaxis minimista, que se ocupa sólo del orden de los constituyentes.

\subsection{Reglas morfológicas del componente de expresión}

Después de ocuparnos de las reglas morfológicas que se manifiestan principalmente en el fondo léxico, pasamos a tratar aquellas reglas morfológicas que operan en el componente de expresión. De nuevo, esto no quiere decir que no deban hacer referencia a la información que proporcionan el fondo léxico y la formación de predicaciones. La formación de predicaciones, por ejemplo, introduce los operadores que dan cuenta de los accidentes verbales:

(22)
a. PAS dedicate $\left(\mathrm{dl}_{\mathrm{v}}: \operatorname{Mary}_{\mathrm{N}}\left(\mathrm{x}_{1}\right)\right)_{\mathrm{Ag} / \mathrm{S} / \mathrm{Top}}$ $\left(d 1 x_{2}: \operatorname{book}_{N}\left(x_{2}\right):\right.$ new $_{A}$ $\left.\left(\mathrm{x}_{2}\right)\right)_{\mathrm{Pac} / \mathrm{O}}\left(\mathrm{dl}_{3}: \operatorname{David}_{\mathrm{N}}\left(\mathrm{x}_{3}\right)\right)_{\text {Ben } / F o c}$

b. Mary dedicated the new book to David. 
Hecha esta salvedad, es necesario llevar a cabo una primera distinción dentro de las reglas del componente de expresión. Las reglas de activación A son aquellas que activan una forma $\mathrm{F}$ y las reglas de colocación son aquellas que dada una forma $\mathrm{F}$ le asignan una posición $\mathrm{P}$ en el orden lineal de la cláusula. En el ejemplo anterior, la regla de activación asigna la forma al pasado del verbo y la regla de colocación asigna al verbo la posición segunda en el orden lineal de la cláusula:
a. Activación:
$\mathbf{R}_{\mathrm{i}}--\mathbf{F}_{\mathbf{j}}$
Pasado[dedicat -$]=$ dedicated
b. Colocación:

$$
\begin{aligned}
& F_{i}---P_{j} \\
& \text { dedicated- }--P_{2}
\end{aligned}
$$

Las reglas de activación son responsables, como mínimo, de los siguientes cambios (Dik 1989: 300):

(i) La reduplicación total o parcial del operando, como en el caso del tema de perfecto en griego:
a. lu-o, «desatar» le-lu-ka, «haber desatado»
b. qu-o, «sacrificar» te-qu-ka, «haber sacrificado»
c. klei-o, «cerrar» ke-klei-ka, «haber cerrado»

(ii) La mutación del operando, como sucede en los verbos fuertes del inglés antiguo del ejemplo (25.a), o el genitivo en alemán en (25.b):
a. risan ras rison «to rise» creopan creap crupon «to creep» faran for foron «to go»
b. der Vater des kleinen Kindes «el padre del niño pequeño»

(iii) La introducción de una forma auxiliar, como un verbo auxiliar:

(26) Los niños estaban dibujando

(iv) La adición de una forma terminal, como un afijo en el ejemplo (27.a) o una adposición, bien se trate de una preposición, como en (27.b) o una postposición, como en (27.c): 
(27)

a. interrogate, interrogation, interrogative, interrogator, etc.

b. ad urbem «ante la ciudad»

c. dem Haus gegenüber «ante la casa»

Varios de estos procesos pueden tener lugar simultáneamente y fenómenos como la liaison en francés, fenómeno ejemplificado en (28.a), o la incorporación sintáctica al complejo verbal del objeto pronominal en inglés, como se recoge en (28.b), indican que las reglas de expresión son interdependientes: ${ }^{7}$
a. petit ami/peti-tami
b. switch off the light
switch it off
*switch off it

El ejemplo (28.a) ilustra la interdependencia entre el orden y la realización fonética, que no puede establecerse hasta que se ha asignado orden a los constituyentes de la cláusula. En el ejemplo (28.b) apreciamos cómo la morfología de los constituyentes depende también del orden; incluso hay casos en los que ésta no puede establecerse hasta que el orden se ha fijado, como en el contexto de un objeto pronominal en inglés, que no puede segregarse del complejo verbal. Otro caso de interdependencia forma-orden ha sido citado por Bakker (1990: 237), quien ha indicado que la forma de la segunda persona del singular del verbo en holandés depende de si éste aparece antes del pronombre, como en (29.a), o después del de dicha categoría, como en (29.b):

(29)

a. Je lacht nooit eens tú ríes nunca una vez «Tú no te ríes nunca»

b. Nooit lach je eens nunca ríes tú una vez «Nunca te ríes tú»

Una traducción al inglés del ejemplo anterior muestra un fenómeno semejante:

${ }^{7}$ Este hecho ha sido destacado por DIK (1989: 290) y BAKKER (1990: 237). 
(30)

a. You never laugh.

b. Never do you laugh.

Junto con las reglas de activación, que son de naturaleza morfológica o fonológica, y las de colocación, de tipo sintáctico, encontramos las reglas de prioridad léxica. Estas reglas están justificadas por el hecho de que ciertas reglas morfologicas que fueron productivas en la diacronía no lo son en la sincronía. Un ejemplo: interrogator no sólo consta de un sufijo muy productivo en inglés moderno (er/or); también se aprecia, en la etimología de la palabra un prefijo, con el que tendríamos que remontarnos al latín para llegar al momento en que interrogo pudo formarse de manera productiva a partir de la base rogo y el prefijo inter. Como una de las reglas que rigen el uso de estos afijos no está disponible en la sincronía del inglés moderno (la de introducción del prefijo inter), el predicado nominal interrogator no puede generarse por medio de las reglas de formación de predicados y debe hacerse disponible por prioridad léxica, lo que quiere decir que el predicado debe tener una entrada independiente en el lexicón, de manera que constituya su propio marco predicativo. Otro ejemplo de regla de prioridad léxica lo constituyen los verbos irregulares en inglés, cuyo pretérito y participio perfecto no pueden formarse por una regla productiva. Las formas irregulares de estos verbos aparecen en el lexicón, en la entrada léxica correspondiente al infinitivo:

go $_{\mathrm{Pas}}-----$ goed $^{* * *}$

goed***_- - - - prioridad léxica- - - - - go $_{\mathrm{v}}$

go $\{$ Pretérico Pasado went, Participio Pasado gone\}

Del ejemplo anterior se deduce que existen, como mínimo, dos tipos de reglas morfológicas. ${ }^{8}$ Las primeras, al igual que la del pasado de los verbos regulares, no activan otras reglas morfológicas, aunque sí fonológicas y alofónicas, lo que implica que tienen reflejo en la expresión lingüística:

(32) Pretérito Pasado[want-] = wanted

${ }^{8}$ De Groot (1990: 190) se refiere a estos dos tipos de reglas como primary triggers y secondary triggers y distingue unos de otros asi: primary triggers are unique in the sense that they correspond to one element, whereas secondary triggers do not have this property. 
Las reglas del otro tipo, como ***, sólo activan la aplicación de otras reglas, como la regla de prioridad léxica. Las reglas del tipo de $* * *$ tienen como única función la activación de otras reglas léxicas o morfológicas pero no fonológicas o alofónicas y carecen, por tanto de reflejo en la expresión lingüística. Tanto unas como otras son reglas de activación, si bien, en el caso de las primeras, la activación es directa y, en el segundo caso, la activación es indirecta. Por esta razón nos referiremos a ellas como reglas de activación directa y reglas de activación indirecta. La regla del pasado irregular mostrada en el ejemplo (31) es una regla típica de activación directa. Como ejemplo de regla de activación indirecta, proponemos la del Paciente, que activa, prototípicamente, la del Acusativo:

(33)

Paciente- - - - Acusativo

Acusativo[Ager] $=$ Agrum

Es de destacar que, una vez que la regla de activación indirecta del Paciente ha activado la regla de Acusativo, esta actúa como una regla de activación directa. Lo mismo sucede con la regla del plural:

Agrum[plural $]=$ Agros

Por último, hay que hacer notar que, aunque hemos descrito la aplicación de dos reglas, la regla de activación indirecta de Paciente no activaría en primer lugar la regla de activación directa de Acusativo y después la de plural, sino que ambas se activarían simultáneamente.

\section{Conclusiones}

En las páginas anteriores hemos recorrido la morfología derivativa y la morfología flexiva de la GF de Dik. En nuestro repaso de la morfología propuesta por el lingüista holandés, hemos examinado los aspectos ya consensuados por el grupo de Amsterdam, al tiempo que hemos dado una interpretación a algunos aspectos todavía por unificar en el campo funcionalista, con lo cual esperamos haber ofrecido una visión, si no exhaustiva, sí global, de las cuestiones que nos han ocupado. Al término de dicho repaso, confiamos en haber ilustrado convenientemente nuestra tesis inicial según la cual la morfología de la GF no está unificada ni es independiente. 
En lo que se refiere a las aportaciones de este trabajo, creemos que éstas pueden resumirse así:

La GF tiene el poder descriptivo suficiente para dar cuenta de la estructura subyacente de demostrativos pertenecientes a sistemas no binarios (como el español este-ese-aquel); los pronombres posesivos en inglés (dada la regla de formación de predicados a partir de términos por medio de la asignación de una función semántica al término de entrada, la función semántica Poseedor asignada a la estructura subyacente de los pronombres personales produce los pronombres posesivos); las estructuras subyacentes de los términos que se obtienen por derivación morfológica (con o sin cambio de categoría léxica del término de entrada); y la elipsis de algunos operandos.

Debe distinguirse entre reglas morfologicas de activación directa, y reglas de activación indirecta. Las primeras tienen reflejo en la expresión lingüística, mientras que las últimas desempeñan únicamente la función de activar la aplicación de otras reglas léxicas o morfológicas pero no fonologicas o alofónicas y carecen, por tanto de reflejo en la expresión lingüística.

\section{REFERENCIAS BIBLIOGRÁFICAS}

ANDERSON, S.R. 1982. "Where is morphology?» Linguistic Inquiry 13: 571-612.

ARONOFF, M. 1976. Word Formation in Generative Grammar. Cambridge, Mass.: MIT Press.

BAKKER, D. 1990. «A formalism for Functional Grammar expression rules» en Connolly y Dik eds.: 45-63.

BAUER, L. 1988. Introducing Linguistic Morphology. Edinburgh: Edinburgh University Press.

Bolkestein et al eds. 1985. Predicates and Terms in Functional Grammar. Dordrecht: Foris.

BROWN, D.R. 1985. «Term operators» en Bolkestein et al eds.: 127-145.

Chomsky, N. 1965. Aspects of the Theory of Syntax Cambridge, Mass.: The MIT Press.

Chомsкy, N. 1981. Lectures on Government and Binding. Dordrecht: Foris.

Chomsky, N. y M. Halle 1968. The Sound Pattern of English. New York: Harper and Row.

Connolly, J. y S.C. Dik, eds. 1990. Functional Grammar and the Computer. Dordrecht: Foris.

DiK, S.C. 1980. Studies in Functional Grammar. London \& New York: Academic Press.

Dik, S.C. 1989. The Theory of Functional Grammar. Part I: The Structure of the Clause. Dordrecht: Foris Publications. 
Downing, A. y P. Locke 1992. A University Course in English Grammar. New York: Prentice Hall.

Givón, T. 1993. English Grammar: A Function-Based Introduction (2 vols.). Amsterdam: John Benjamins.

GroOt, C. DE. 1990. «Morphology and the typology of expression rules» en Hannay y Vester eds.: 187-201.

HANNAY, M. y E. VESTER eds. 1990. Working with Functional Grammar: Descriptive and Computational Applications. Dordrecht: Foris.

HeNGeveld, K. 1990. «The Hierarchical Structure of Utterances» en Nuyts et al. eds. $1-23$.

HocketT, C. 1954. «Two Models of Grammatical Description». Word 10: 210-231.

IngemanN, F. ed. 1983. Proceedings of the 1982 Mid-America Linguistics Conference. University of Kansas.

Katamba, F. 1993. Morphology. London: MacMillan.

KIPARSKY, P. 1982. Explanation in Phonology. Dordrecht: Foris.

KIPARSKY, P. 1983. «Word Formation and the Lexicon» en Ingemann ed.: 73-129.

MARLE, J. VAN 1985. On the Paradigmatic Dimension of Morphological Creativity. Dordrecht: Foris.

MatTHEws, P.H. 1972. Inflectional Morphology. Cambridge: Cambridge University Press.

MatThews, P.H. 1974. Morphology. Cambridge: Cambridge University Press.

MATTHEWs, P.H. 1994. Grammatical theory in the United States from Bloomfield to Chomsky. Cambridge: Cambridge University Press.

Moreno CABreRA, J.C. 1991. Curso universitario de lingütstica general I: Teoría de la gramática y sintaxis general. Madrid: Síntesis.

NidA, E.A. 1970. Morphology. The University of Michigan Press.

NuTTs, J. at al eds. 1990. Layers and Levels of Representation in Language Theory. A Functional View. Amsterdam: John Benjamins.

RukнoFf, J. 1990. «Towards a Unified Analysis of Terms and Predications» en Nuyts et al eds.: 165-188.

RoBINs, R.H. 1959. «In Defence of WP» (reimpreso en Robins, R. 1970. Diversions of Bloomsbury. Amsterdam: North Holland).

SCALISSE, S. 1987. Morfología generativa. Madrid: Alianza Editorial.

SIEGEL, D. 1979. Topics in English Morphology. New York and London: Garland Publishing.

SPENCER, A. 1992. Morphological Theory. London: Blackwell.

VARELA Ortega, S. 1990. Fundamentos de Morfología. Madrid: Síntesis.

VoOgt-VAN ZUTPHEN, H. 1990. «Towards a Lexicon of Functional Grammar» en Conolly y Dik eds.: 151-176.

WATTERS, J.R. 1985. «The place of morphology in Functional Grammar: the case of the Ejagham verb system» en Bolkestein et al eds.: 85-103. 